$\xi_{p}=-$

\title{
Stability of the equilibrium points in the circular restricted four body problem with oblate primary and variable mass
}

\author{
Abdullah Abduljabar Ansari * \\ Mathematics Department, Majmaah University, College of Science, Al-Zulfi, KSA \\ *Corresponding author E-mail: a.ansari@mu.edu.sa
}

\begin{abstract}
This paper investigates the liberation points and stability of the restricted four body problem with one of the primaries as oblate body and the infinitesimal body is taken as variable mass. Due to oblateness, the equilateral triangular configuration is no longer exists and becomes an isosceles triangular configuration. Moreover, we have found seven equilibrium points out of which three are asymptotically stable (dark black in the tables) and rest four are unstable.
\end{abstract}

Keywords: Asymptotically Stable; Circular Restricted Four Body Problem; Isosceles Triangular Configuration; Oblateness Factor; Variable Mass.

\section{Introduction}

Many researchers have studied about the stability of the equilibrium points in the restricted problems. Moulton [12] studied the four bodies in which three bodies of spherical mass are placed at the vertices of an equilateral triangle. Szebehely [18] explained in detail in his book about the stability of restricted problems. Bhatnagar [3] studied the periodic orbits of collision in the plane circular problem of four bodies and all the primaries are spherical in shape, placed at the vertices of an equilateral triangle. Khanna [6] studied the existence and stability of libration points in the restricted three body problem where the smaller primary is a triaxial rigid body and the bigger one an oblate body. They found five libration points, in which three are collinear unstable points and two are triangular stable points and these stable points have short and long periodic orbits. Douskos [4] found the existence of nonplanar equilibrium points in the three-dimensional restricted threebody problem with oblateness. Mittal [11] have studied the periodic orbits generated by Lagrangian solutions of the restricted three body problem when one of the primaries is an oblate body and shown the effect of oblateness on the periodic orbits. Baltagiannis [2] have investigated the equilibrium points and found eight liberation points which are unstable in the restricted four body problem where the primaries are spherical in shape and placed at the vertices of an equilateral triangle. Md Chand Asique [8], [9] studied about restricted four body problems once by taking one of the primaries as oblate and in another by taking primaries as photogravitational and oblate or prolate. And found that there exist eight liberation points in which three are stable and five are unstable except for some values of the mass parameter.

Researchers have also studied about restricted three and four body problem with variable mass ie. Shrivastava [13], Singh [14], [15], [16], [17], Zhang [19], Abouelmagd [1].

After reviewing all the literature, we impressed to do the work on the restricted four body problem with one of the primaries as oblate body and all the three primaries are placed at the vertices of a triangle and also the infinitesimal mass is taken as variable mass.
The entire study has been done in various sections. In the first section, we have determined the mean motion and shown that when we replace one primary as an oblate body, the triangular configuration becomes isosceles instead of equilateral as in the classical case (Bhatnagar [3]). In the second section, we have found the equations of motion in the Cartesian form. In the third section, we have found the equilibrium points. In the fourth section, we have actually shown the stability of equilibrium points. In the last section, we have concluded the problem.

\section{Equations of motion}

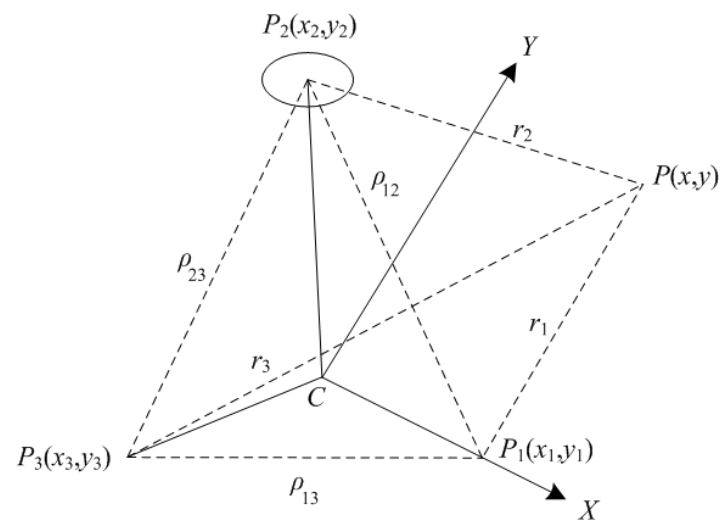

Fig. 1: Isosceles Triangular Configuration of the Primaries $\mathrm{P}_{1} \mathrm{P}_{2} \mathrm{P}_{3}$.

Let $\mathrm{P}_{1}, \mathrm{P}_{2}$ and $\mathrm{P}_{3}$ be three bodies of masses $\mathrm{m}_{1}, \mathrm{~m}_{2}$ and $\mathrm{m}_{3}$ moving in their mutual gravitational field in circular orbits around their center of mass $\mathrm{C}$ which is the geometric center of the triangular configuration $\mathrm{P}_{1} \mathrm{P}_{2} \mathrm{P}_{3}$. Here the body $\mathrm{P}_{2}$ is an oblate body and others are spherical in shape. And let $\mathrm{P}_{1} \mathrm{P}_{2}=\rho_{12}, \mathrm{P}_{2} \mathrm{P}_{3}=\rho_{23}$ and $\mathrm{P}_{1} \mathrm{P}_{3}$ $=\rho_{13}$. And let the co-ordinates of $P_{i}$ be $\left(X_{i}, Y_{i}\right)(i=1,2,3)$. The center of mass can be regarded at rest by neglecting the external forces. If oblateness is ignored then equilateral configuration $\mathrm{P}_{1} \mathrm{P}_{2} \mathrm{P}_{3}$ is a solution and we have $\mathrm{n}^{2} \ell^{3}=\mathrm{G}\left(\mathrm{m}_{1}+\mathrm{m}_{2}+\mathrm{m}_{3}\right)$ (Mccuskey 
[7]), where $\ell$ is the length of the side of the equilateral triangle $\mathrm{P}_{1} \mathrm{P}_{2} \mathrm{P}_{3}, \mathrm{n}$ is the mean motion and $\mathrm{G}$ is the gravitational constant.

Due to oblateness factor $\sigma$ ' equilateral triangular configuration will no longer exist. Let us suppose, in our case, $\rho_{12}=\ell+\lambda_{1}, \rho_{23}=\ell+\lambda_{2}$, $\rho_{13}=\ell+\lambda_{3}$ where $\lambda_{1}, \lambda_{2}$ and $\lambda_{3}<1$. Proceeding as in Mccuskey [7], we get the following six equations in the Cartesian form

$$
\begin{aligned}
& \left(-\mathrm{n}^{2}+\frac{\mathrm{G} \cdot \mathrm{m}_{3}}{\left(\ell+\lambda_{3}\right)^{3}}+\frac{\mathrm{G} \cdot \mathrm{m}_{2}}{\left(\ell+\lambda_{1}\right)^{3}}+\frac{3 \mathrm{G} \cdot \mathrm{m}_{2} \cdot \sigma^{\prime}}{2\left(\ell+\lambda_{1}\right)^{5}}\right) \cdot \mathrm{X}_{1}+ \\
& \left(-\frac{\mathrm{G} \cdot \mathrm{m}_{2}}{\left(\ell+\lambda_{1}\right)^{3}}-\frac{3 \mathrm{G} \cdot \mathrm{m}_{2} \cdot \sigma^{\prime}}{2\left(\ell+\lambda_{1}\right)^{5}}\right) \cdot \mathrm{X}_{2}+\left(-\frac{\mathrm{G} \cdot \mathrm{m}_{3}}{\left(\ell+\lambda_{3}\right)^{3}}\right) \cdot \mathrm{X}_{3}=0, \\
& -\frac{\mathrm{G} \cdot \mathrm{m}_{1}}{\left(\ell+\lambda_{3}\right)^{3}} \mathrm{X}_{1}+\left(-\frac{\mathrm{G} \cdot \mathrm{m}_{2}}{\left(\ell+\lambda_{2}\right)^{3}}-\frac{3 \mathrm{G} \cdot \mathrm{m}_{2} \cdot \sigma^{\prime}}{2\left(\ell+\lambda_{2}\right)^{5}}\right) \cdot \mathrm{X}_{2} \\
& +\left(-\mathrm{n}^{2}+\frac{\mathrm{G} \cdot \mathrm{m}_{1}}{\left(\ell+\lambda_{3}\right)^{3}}+\frac{\mathrm{G} \cdot \mathrm{m}_{2}}{\left(\ell+\lambda_{2}\right)^{3}}+\frac{3 \mathrm{G} \cdot \mathrm{m}_{2} \cdot \sigma^{\prime}}{2\left(\ell+\lambda_{2}\right)^{5}}\right) \cdot \mathrm{X}_{3}=0,
\end{aligned}
$$

$\mathrm{m}_{1} \cdot \mathrm{X}_{1}+\mathrm{m}_{2} \cdot \mathrm{X}_{2}+\mathrm{m}_{3} \cdot \mathrm{X}_{3}=0$

$$
\begin{aligned}
& \left(-\mathrm{n}^{2}+\frac{\mathrm{G} \cdot \mathrm{m}_{3}}{\left(\ell+\lambda_{3}\right)^{3}}+\frac{\mathrm{G} \cdot \mathrm{m}_{2}}{\left(\ell+\lambda_{1}\right)^{3}}+\frac{3 \mathrm{G} \cdot \mathrm{m}_{2} \cdot \sigma^{\prime}}{2\left(\ell+\lambda_{1}\right)^{5}}\right) \cdot \mathrm{Y}_{1}+ \\
& \left(-\frac{\mathrm{G} \cdot \mathrm{m}_{2}}{\left(\ell+\lambda_{1}\right)^{3}}-\frac{3 \mathrm{G} \cdot \mathrm{m}_{2} \cdot \sigma^{\prime}}{2\left(\ell+\lambda_{1}\right)^{5}}\right) \cdot \mathrm{Y}_{2}+\left(-\frac{\mathrm{G} \cdot \mathrm{m}_{3}}{\left(\ell+\lambda_{3}\right)^{3}}\right) \cdot \mathrm{Y}_{3}=0, \\
& -\frac{\mathrm{G} \cdot \mathrm{m}_{1}}{\left(\ell+\lambda_{3}\right)^{3}} \mathrm{Y}_{1}+\left(-\frac{\mathrm{G} \cdot \mathrm{m}_{2}}{\left(\ell+\lambda_{2}\right)^{3}}-\frac{3 \mathrm{G} \cdot \mathrm{m}_{2} \cdot \sigma^{\prime}}{2\left(\ell+\lambda_{2}\right)^{5}}\right) \cdot \mathrm{Y}_{2}+ \\
& \left(-\mathrm{n}^{2}+\frac{\mathrm{G} \cdot \mathrm{m}_{1}}{\left(\ell+\lambda_{3}\right)^{3}}+\frac{\mathrm{G} \cdot \mathrm{m}_{2}}{\left(\ell+\lambda_{2}\right)^{3}}+\frac{3 \mathrm{G} \cdot \mathrm{m}_{2} \cdot \sigma^{\prime}}{2\left(\ell+\lambda_{2}\right)^{5}}\right) \cdot \mathrm{Y}_{3}=0,
\end{aligned}
$$$$
m_{1} \cdot Y_{1}+m_{2} \cdot Y_{2}+m_{3} \cdot Y_{3}=0
$$

These equations will have non-trivial solution, if

$$
\lambda_{1}-\lambda_{3}=\frac{\sigma}{2 \ell}, \quad \lambda_{1}=\lambda_{2}=\lambda \text { (say), }
$$

And

$$
\mathrm{n}^{2}=\frac{\mathrm{G}\left(\mathrm{m}_{1}+\mathrm{m}_{2}+\mathrm{m}_{3}\right)}{\ell^{3}}\left[1-\frac{3}{\ell}\left(\lambda-\frac{\dot{\sigma}}{2 \ell}\right)\right] .
$$

This means that the three bodies remain at the vertices of an isosceles triangle instead of an equilateral triangle as in the classical case.

We shall follow the procedure of Abouelmagd [1]. The equations of motion for the infinitesimal variable mass when the variation of mass is non-isotropic and originating from one point are

$$
\begin{aligned}
& \ddot{X}+\frac{\dot{m}}{m} \dot{X}=-\frac{G m_{1}\left(X-X_{1}\right)}{r_{1}^{3}}-\frac{G m_{2}\left(X-X_{2}\right)}{r_{2}^{3}}- \\
& \frac{3 G m_{2} \sigma^{\prime}\left(X-X_{2}\right)}{2 r_{2}^{5}}-\frac{G m_{3}\left(X-X_{3}\right)}{r_{3}^{3}}, \\
& \ddot{Y}+\frac{\dot{m}}{m} \dot{Y}=-\frac{G m_{1}\left(Y-Y_{1}\right)}{r_{1}^{3}}-\frac{G m_{2}\left(Y-Y_{2}\right)}{r_{2}^{3}}- \\
& \frac{3 G m_{2} \sigma^{\prime}\left(Y-Y_{2}\right)}{2 r_{2}^{5}}-\frac{G m_{3}\left(Y-Y_{3}\right)}{r_{3}^{3}},
\end{aligned}
$$

Where,

$$
\begin{aligned}
& r_{1}^{2}=\left(X-X_{1}\right)^{2}+\left(Y-Y_{1}\right)^{2}, \\
& r_{2}^{2}=\left(X-X_{2}\right)^{2}+\left(Y-Y_{2}\right)^{2}, \\
& r_{3}^{2}=\left(X-X_{3}\right)^{2}+\left(Y-Y_{3}\right)^{2} .
\end{aligned}
$$

Let the co-ordinates of $\mathrm{P}_{\mathrm{i}}$ be $\left(\mathrm{x}_{\mathrm{i}}, \mathrm{y}_{\mathrm{i}}\right)$ and $\mathrm{P}$ be $(\mathrm{x}, \mathrm{y})$ in the rotating system with angular velocity $\mathrm{n}$. Let $\ell$ be the unit of length and $\left(m_{1}+m_{2}+m_{3}\right)$, the unit of mass and the unit of time is so chosen so as to make $G=1$. Using the relation between the inertial and rotating coordinates (Abouelmagd [1]), the equations of motion in a rotating coordinate system for an infinitesimal variable mass $\mathrm{P}$ will be

$$
\begin{aligned}
& \dot{\mathrm{m}}(\dot{\mathrm{x}}-\mathrm{ny})+\mathrm{m}(\ddot{\mathrm{x}}-2 \mathrm{n} \dot{\mathrm{y}})=-\mathrm{U}_{\mathrm{x}} \\
& \dot{\mathrm{m}}(\dot{\mathrm{y}}+\mathrm{nx})+\mathrm{m}(\ddot{\mathrm{y}}+2 \mathrm{n} \dot{\mathrm{x}})=-U_{y}
\end{aligned}
$$

Where

$$
\begin{aligned}
& \mathrm{U}=\frac{\mathrm{n}^{2}}{2}\left(\mathrm{x}^{2}+\mathrm{y}^{2}\right)+\frac{\mu_{1}}{\mathrm{r}_{1}}+\frac{\mu_{2}}{\mathrm{r}_{2}}+\frac{\mu_{3}}{\mathrm{r}_{3}}+\frac{\mu_{2} \sigma}{2 \mathrm{r}_{2}^{3}}, \\
& \mathrm{r}_{\mathrm{i}}^{2}=\left(\mathrm{x}-\mathrm{x}_{\mathrm{i}}\right)^{2}+\left(\mathrm{y}-\mathrm{y}_{\mathrm{i}}\right)^{2}, \\
& \mu_{\mathrm{i}}=\frac{\mathrm{m}_{\mathrm{i}}}{\mathrm{m}},(\mathrm{i}=1,2,3) \\
& \sigma=\frac{\sigma^{\prime}}{\ell^{2}}=\frac{\mathrm{a}^{2}-\mathrm{c}^{2}}{5}, \\
& \mathrm{n}^{2}=\left[1-3\left(\lambda-\frac{\sigma}{2}\right)\right] .
\end{aligned}
$$

$\mu_{1}=\mu, \mu_{2}=1-\mu_{1}-\mu_{3}, \mu_{3}=\alpha_{2} \mu, \alpha_{2}<<1$,

$\left(\mathrm{x}_{1}, \mathrm{y}_{1}\right)=\left(\frac{1}{\sqrt{3}}\left(1+\frac{\lambda}{3}\right), 0\right)$

$\left(\mathrm{x}_{2}, \mathrm{y}_{2}\right)=\left(-\frac{1}{2 \sqrt{3}}\left(1+\frac{7 \lambda}{3}\right), \frac{1}{2}(1+\lambda)\right)$

$\left(\mathrm{x}_{3}, \mathrm{y}_{3}\right)=\left(-\frac{1}{2 \sqrt{3}}\left(1-\frac{5 \lambda}{3}\right),-\frac{1}{2}(1+\lambda)\right)$.

Jacobi integral is

$\mathrm{C}=\frac{1}{2}\left(\dot{\mathrm{x}}^{2}+\dot{\mathrm{y}}^{2}\right)-\frac{\mathrm{n}^{2}}{2}\left(\mathrm{x}^{2}+\mathrm{y}^{2}\right)-\frac{\mu_{1}}{\mathrm{r}_{1}}-\frac{\mu_{2}}{\mathrm{r}_{2}}-\frac{\mu_{3}}{\mathrm{r}_{3}}-\frac{\mu_{2} \sigma}{2 \mathrm{r}_{2}^{3}}$.

According to Jeans' (1928),

$$
\frac{\mathrm{dm}}{\mathrm{dt}}=-\alpha \mathrm{m}^{\beta}
$$

Where $\alpha$ is constant coefficient and $\beta$ is within the limits $0.4 \leq \beta \leq 4.4$ for the stars of the main sequence. While for the rocket $\beta=1$, the mass of the rocket $\mathrm{m}=\mathrm{m}_{0} \mathrm{e}^{-\alpha \mathrm{t}}$ varies exponentially. Where $m_{0}$ is the mass of the infinitesimal body when $t=0$.

To simplify the equations of motion of the variable mass, we use the space-time transformation of Mishcherskii [10] which preserve the dimension of the space and time 


$$
\begin{aligned}
& \mathrm{x}=\gamma^{-\mathrm{q}_{\xi}}, \mathrm{y}=\gamma^{-\mathrm{q}_{\eta}, \mathrm{dt}}=\gamma^{-\mathrm{k}} \mathrm{d} \Gamma, \ell_{1}=
\end{aligned}
$$

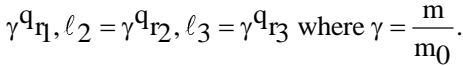

After using equations (5) and (6) in equation (3) and taking $q=1 / 2, \beta=1, k=0$, the equations of motion (3) become

$\xi^{\prime \prime}-2 \mathrm{n} \eta^{\prime}=\frac{\partial \mathrm{w}}{\partial \xi}$,

$\eta^{\prime \prime}+2 \mathrm{n} \xi^{\prime}=\frac{\partial \mathrm{w}}{\partial \eta}$

Where, $w=\left(\frac{n^{2}}{2}+\frac{\alpha^{2}}{8}\right)\left(\xi^{2}+\eta^{2}\right)+\gamma^{\frac{3}{2}}\left[\frac{\mu_{1}}{\ell_{1}}+\frac{\mu_{2}}{\ell_{2}}+\frac{\mu_{3}}{\ell_{3}}+\frac{\mu_{2} \sigma}{2 \ell_{2}^{3}}\right]$,

$\ell_{i}^{2}=\left(\xi-\xi_{i}\right)^{2}+\left(\eta-\eta_{i}\right)^{2}, \quad i=1,2,3$.

\section{Liberation points}

The libration points with variable mass $\mathrm{m}$ are obtained from the solution of the equations:

$$
\mathrm{w}_{\xi}=0, \mathrm{w}_{\eta}=0,
$$

i.e.

$$
\left(n^{2}+\frac{\alpha_{1}^{2}}{4}\right) \xi-\gamma^{3 / 2}\left[\begin{array}{c}
\frac{\mu_{1}\left(\xi-\xi_{1}\right)}{\ell_{1}^{3}}+\frac{\mu_{2}\left(\xi-\xi_{2}\right)}{\ell_{2}{ }^{3}}+ \\
\frac{\mu_{3}\left(\xi-\xi_{3}\right)}{\ell_{3}^{3}}+\frac{3 \mu_{2} \sigma\left(\xi-\xi_{2}\right)}{\ell_{2}{ }^{5}}
\end{array}\right]=0,
$$$$
\left(n^{2}+\frac{\alpha_{1}^{2}}{4}\right) \zeta-\gamma^{3 / 2}\left(\begin{array}{l}
\frac{\mu_{1} \eta}{\ell_{1}^{3}}+\frac{\mu_{2}\left(\eta-\eta_{2}\right)}{\ell_{2}{ }^{3}}+ \\
\frac{\mu_{3}\left(\eta-\eta_{3}\right)}{\ell_{3}{ }^{3}}+\frac{3 \mu_{2} \sigma\left(\eta-\eta_{2}\right)}{\ell_{2}{ }^{5}}
\end{array}\right)=0 .
$$

If we take $\alpha_{1}=0$ or $\gamma=1$ in equations (8), we will obtain the equations of Md Chand Asique [8].

For $\alpha_{1} \neq 0$, we determine the positions of libration points numerically for different values of $\alpha_{1}, \gamma$ and $\sigma$. We have used the value of $\mu=0.019$ throughout the paper. We have determined the locations of the libration points for $0 \leq \alpha_{1} \leq 1,0<\gamma \leq 1$ and $\sigma$ in the plane of

\begin{tabular}{|c|c|}
\hline$\alpha_{1}$ & Location of non-collinear libration points $\left(\xi_{0}, \eta_{0}\right)$ \\
\hline 0.2 & $\begin{array}{l}\text { (-0. 2844547475354861, 0. 4873932611465871), (-0. 2844419450686945, 0. 48739396303304383), } \\
(0.2956270600811379,0.06192302747859898) \\
(0.29556491099304,0.061977680344770456) \\
(-\mathbf{0 . 1 3 5 0 3 4 7 5 8 7 6 9 8 7 1 2 5 , - 0 . 2 2 5 1 7 3 6 0 6 7 6 1 7 6 0 3 2 )} \\
(0.08910163326559607,-0.1947940210736529) \\
(0.08858893531830736,-0.19497567534431656)\end{array}$ \\
\hline 0.4 & 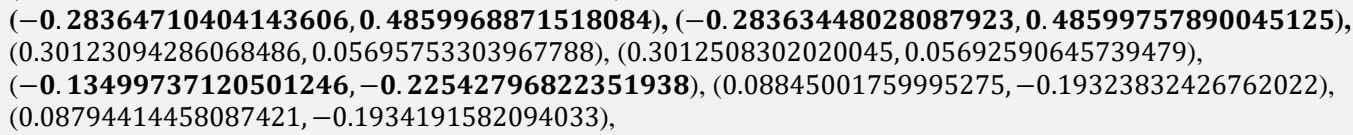 \\
\hline 0.6 & 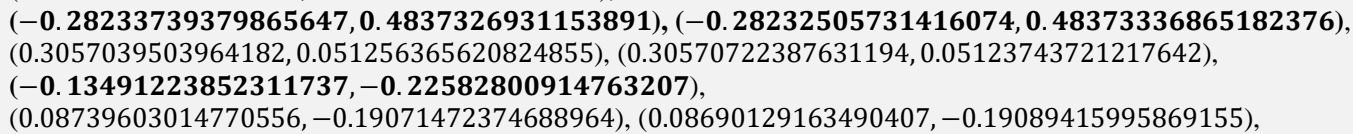 \\
\hline 0.8 & $\begin{array}{l}(-\mathbf{0 . 2 8 0 5 7 5 8 7 7 4 8 2 3 7 7 5 6 , 0 . 4 8 0 6 8 7 8 7 3 7 0 1 1 0 0 2}),(-\mathbf{0 . 2 8 0 5 6 3 9 2 2 4 3 1 4 2 8 1 , 0 . 4 8 0 6 8 8 5 2 7 8 7 2 9 2 4 9 3 )} \\
(0.30870711000440193,0.0455491002517442) \\
(0.3087036897405585,0.045537506676822055) \\
(-\mathbf{0 . 1 3 4 7 5 4 4 2 0 3 1 9 8 6 8 1 7 , - 0 . 2 2 6 3 3 5 3 1 1 4 3 4 0 2 3 6 3 )} \\
(0.08598360773051841,-0.18731918845451875) \\
(0.08550395792282305,-0.18749661146118424)\end{array}$ \\
\hline 1 & $\begin{array}{l}(-0.27842529761978424,0.47697124596666035),(-0.278413800513326,0.4769718747311898), \\
(0.31045438312395973,0.04011885700185765),(0.3104482254377997,0.04011185335496099), \\
(-0.13450560314601656,-0.22689686342456628),(0.08426697766961469,-0.1831720152674377), \\
(0.08380588756172369,-0.1833467703805845)\end{array}$ \\
\hline
\end{tabular}
motion of the primaries i.e. in $\xi \eta$-plane (Tables 1 to 5).

Table 1: Equilibrium Points on $\xi \eta-$ plane when $\gamma=0.2, \mu=0.019, \mu_{1}=\mu_{,} \mu_{2}=1-\mu_{1}-\mu_{3}, \mu_{3}=\alpha_{2} \mu, \alpha_{2}=0.01, \lambda=0.01, \sigma=0.01$

Table 2: Equilibrium Points on $\xi \eta-P l a n e$ when $\gamma=0.2, \mu=0.019, \mu_{1}=\mu, \mu_{2}=1-\mu_{1}-\mu_{3}, \mu_{3}=\alpha_{2} \mu, \alpha_{2}=0.01, \lambda=0.01, \sigma=0.001$.

$\alpha_{1} \quad$ Location of non-collinear libration points $\left(\xi_{0}, \eta_{0}\right)$

(-0.27737428196671116, 0.4753141375042226), (-0.27736088039910345, 0.47531430772230054),

$(0.30816532401901964,0.05085949009441141),(0.3081658127439091,0.050842092067477716)$,

$0.2 \quad(-0.13512757732932743,-0.2259848782311532),(0.08626922845525134,-0.19074821913640916)$, ( $0.08574318848459206,-0.19093498831074399)$

$(-0.276514529057806,0.4738279841922493),(-0.27650131557941987,0.47382814636694276)$,

$0.4 \quad(0.30951013516759607,0.04823866135883527),(0.309507637396958,0.04822463529653216)$,

(-0.1350525369799976, - 0. 22622371438775393), (0.08560814447201556, - 0.1891519510249989),

$(0.08508947825670105,-0.18933789092736564)$

(-0.27512023435144156, 0.4714180795731819),

(-0.2751073231967866, 0.47141822902146924),

$0.6 \quad(0.31098755644015535,0.044535802991353214),(0.31098235305115085,0.0445255691047567)$,

(-0.13491104015014793, - 0. 22659193767379085), (0.08453951118106617, -0.1865637218612278),

$(0.08403286380478125,-0.18674824135078452)$

$(-0.2732447690488095,0.4681770031793249),(-0.2732322592447567,0.4681771361263173)$,

$0.8 \quad(0.3121152226703554,0.04030988922977912),(0.31210831853721316,0.04030299467544001)$,

(-0.13468797983082983, - 0. 22704647475537315), (0.0831087434364661, -0.18308356161055264),

$(0.08261835953788292,-0.18326602140761192)$ 
(-0.2709547286676093, 0. 4642202392388929), (-0.27094270063732967, 0. 46422035298957504),

$(0.3126851670827154,0.0359679666676881),(0.31267749177692405,0.035963718032039196)$,

$1 \quad(-0.1343770990885276,-0.22753423562722308),(0.08137175743303554,-0.17883671147586827)$ $(0.08090133741343,-0.17901642195007444)$

Table 3: Equilibrium Points on $\xi \eta-P l a n e$ When $\gamma=0.9, \mu=0.019, \mu_{1}=\mu, \mu_{2}=1-\mu_{1}-\mu_{3}, \mu_{3}=\alpha_{2} \mu, \alpha_{2}=0.01, \lambda=0.01, \sigma=0.01$.

$\alpha_{1} \quad$ Location of non-collinear libration points $\left(\xi_{0}, \eta_{0}\right)$

$(-0.5893191553255784,1.0098309193829864),(-0.5892912406858347,1.0098314710184342)$,

$0.2 \quad(0.6543416427541445,0.10557889744898512),(0.6543402761354244,0.10554493133265302)$,

(-0. 28652280922120615, - 0. 4795502733234415), (0.18276459531243183, -0.4032321451552517),

$(0.1816646386312163,-0.40362544458021715)$

(-0.587543806281089, 1. 006762110438087), (-0.5875162795843681, 1. 0067626451897913),

$(0.6568815071071648,0.10031397498284132),(0.656874832530823,0.1002863412924406)$,

$0.4 \quad(-0.28636035319206316,-0.4800412553955047)$,

$(0.18138996818041034,-0.399912214358862)$

$(0.18030528716801575,-0.4003037284170671)$

$(-0.5846639528937032,1.0017845938892886),(-0.584637050002617,1.0017851018766253)$,

$0.6 \quad(0.6596970356669626,0.09281654710236555),(0.6596854314302416,0.09279619898446437)$,

(-0. 28605738141142484, - 0. 48079780806076533), (0.17916686123637268, - 0.3945273061997747), $(0.1781070772342044,-0.394915770249845)$

(-0.5807889416010946, 0.9950880367192488), $(-0.5807628673147037,0.9950885099033113)$, $(0.6618492348608215,0.0841966822217591),(0.6618344778793793,0.0841828810016387)$,

$0.8 \quad(-\mathbf{0 . 2 8 5 5 8 4 6 6 3 0 4 4 8 6 1 8 5 , - 0 . 4 8 1 7 3 1 4 0 6 8 1 4 8 0 6 9 )},(0.17618839860357202,-0.3872830529399044)$, $(0.17516230299794355,-0.387667109442528)$

(-0.5760553930422728, 0.9869093445552544), (-0.5760303140987504, 0.9869097770924614),

$1 \quad(0.6629075476206654,0.07528640928944615),(0.6628913679915919,0.07527787545257904)$,

(-0.28493088572833014, - 0. 48273354109923533), (0.17256943660416726, -0.3784372676094282) $(0.1715846956777738,-0.37881546313938497)$

Table 4: Equilibrium Points on $\xi \eta-P l a n e$ When $\gamma=0.9, \mu=0.019, \mu_{1}=\mu, \mu_{2}=1-\mu_{1}-\mu_{3}, \mu_{3}=\alpha_{2} \mu, \alpha_{2}=0.01, \lambda=0.01, \sigma=0.001$.

$\alpha_{1} \quad$ Location of non-collinear libration points $\left(\xi_{0}, \eta_{0}\right)$

(-0.5867147930548995,1.0054152189225916), (-0.5866862572900899, 1. 0054154364034669),

0.2

$(0.6551354551727054,0.10603336480157897),(0.6551337739982154,0.1059991438760604)$,

$(0.18234640768312535,-0.40358207835360954),(0.18122926468225514,-0.40397912735471814)$,

(-0.28664173579815866, -0.47957767663338186$)$

(-0.5848802483480661, 1.0022441981066375), (-0.5848521124212047, 1. 0022443986705354),

$(0.6576809166299544,0.10070459997768906),(0.6576739111674842,0.10067677957824271)$,

$(0.1809446848987448,-0.4001934077937596),(0.17984327263298627,-0.400588693436064)$,

$(-0.28647472579765515,-0.4800779909956699)$

(-0.5819048189777963,0.9971016828960256), (-0.5818773257478929, 0.9971018567108072),

$0.6 \quad(0.6604935559125026,0.09311774879779251),(0.6604816133123983,0.09309729819691485)$,

(-0. 28616337235961264, - 0. 4808478420522158), (0.1786788544500434, -0.39469898977904483),

$(0.17760307969736366,-0.3950912540956996)$

(-0.5779020144162265, 0.9901845672970899), (-0.5778753744407822, 0.9901847064635233),

$0.8 \quad(0.6626266774066116,0.08439977435583709),(0.6626115929440162,0.0843859477878872)$,

(-0. 28567810823813344, - 0. 48179570244491715), (0.17564525508145484, -0.38731125901657965),

$(0.1746041664248091,-0.3876991404576824)$

(-0.5730134848896092, 0.9817384845518321), (-0.5729878692088847, 0.9817385834687645),

$(0.6636482836538359,0.0753959729678519),(0.6636318037586589,0.07538747280245163)$,

(-0. 28500827262358247, - 0. 4828098979811156), (0.17196249382009984, -0.3782961989988564),

$(0.1709639738830179,-0.3786782276598948)$

Table 5: Equilibrium Points on $\xi \eta-P l a n e$ when $\gamma=1, \alpha_{1}=0, \mu=0.019, \mu_{1}=\mu, \mu_{2}=1-\mu_{1}-\mu_{3}, \mu_{3}=\alpha_{2} \mu, \alpha_{2}=0.01, \lambda=0.01, \sigma=0.001$.

Location of non-collinear libration points $\left(\xi_{0}, \eta_{0}\right)$

(-0.6190538213932957,1.0608425744823706), (-0.6190235956420039, 1.0608428053417385),

(0.6895070565950088, 0.11376132383059608), (0.689507793369561, 0.11372253745325724),

(0.19269031313367624, -0.42658833480963965$),(0.1915071141652341,-0.4270074972886581)$,

$(-0.30220126869778274,-0.5053424947706442)$

\section{Stability of the liberation points}

The linear stability of the libration points has been determined by giving the displacement to $\left(\xi_{0}, \eta_{0}\right)$ as

$\xi=\xi_{0}+\mathrm{u}, \eta=\eta_{0}+\mathrm{v},(\mathrm{u}, \mathrm{v})<<1$

Where $\left(\xi_{0}, \eta_{0}\right)$ is the libration point for a fixed value of time $t$ ?

From the equations (5) and (9), we obtain the variational equations

as

$\ddot{\mathrm{u}}-2 \mathrm{n} \dot{\mathrm{v}}=(\mathrm{w} \xi \xi) 0 \mathrm{u}+(\mathrm{w} \xi \eta)_{0} \mathrm{v}$, $\ddot{\mathrm{v}}+2 \mathrm{n} \dot{\mathrm{u}}=\left(\mathrm{w}_{\eta \xi}\right)_{0} \mathrm{u}+\left(\mathrm{w}_{\eta} \eta\right)_{0} \mathrm{v}$

Where the subscript ' 0 ' in equations (10) represents that the values are to be found at the libration point $\left(\xi_{0}, \eta_{0}\right)$ under consideration. We have 


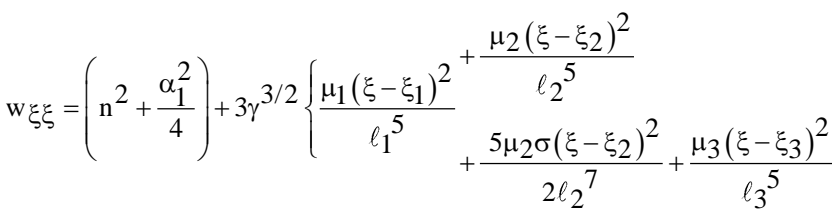

$$
\begin{aligned}
& -\gamma^{3 / 2}\left(\frac{\mu_{1}}{\ell_{1}^{3}}+\frac{\mu_{2}}{\ell_{2}{ }^{3}}+\frac{3 \mu_{2} \sigma}{2 \ell_{2}{ }^{5}}+\frac{\mu_{3}}{\ell_{3}^{3}}\right), \\
& \mathrm{w}_{\eta \xi}=\mathrm{w}_{\xi \eta}=3 \gamma^{3 / 2}\left(\frac{\mu_{1}\left(\xi-\xi_{1}\right) \eta}{\ell_{1}^{5}}+\right. \\
& \frac{\mu_{2}\left(\xi-\xi_{2}\right)\left(\eta-\eta_{2}\right)}{\ell_{2}{ }^{5}}+\frac{5 \mu_{2} \sigma\left(\xi-\xi_{2}\right)\left(\eta-\eta_{2}\right)}{\ell_{2}{ }^{7}} \\
& \left.+\frac{\mu_{3}\left(\xi-\xi_{3}\right)\left(\eta-\eta_{3}\right)}{\ell_{3}^{5}}\right), \\
& \mathrm{w}_{\eta \eta}=\left(\mathrm{n}^{2}+\frac{\alpha_{1}^{2}}{4}\right)+3 \gamma^{3 / 2}\left\{\begin{array}{l}
\frac{\mu_{1} \eta^{2}}{\ell_{1}^{5}}+\frac{\mu_{2}\left(\eta-\eta_{2}\right)^{2}}{\ell_{2}^{5}} \\
+\frac{5 \mu_{2} \sigma\left(\eta-\eta_{2}\right)^{2}}{2 \ell_{2}^{7}}+\frac{\mu_{3}\left(\eta-\eta_{3}\right)^{2}}{\ell_{3}^{5}}
\end{array}\right\} \\
& -\gamma^{3 / 2}\left(\frac{\mu_{1}}{\ell_{1}^{3}}+\frac{\mu_{2}}{\ell_{2}{ }^{3}}+\frac{3 \mu_{2}}{2 \ell_{2}{ }^{5}}+\frac{\mu_{3}}{\ell_{3}^{3}}\right),
\end{aligned}
$$

If we take $\alpha_{1}=0$, the system (10) reduced to a system with constant mass. For $\alpha_{1}>0$, the coordinates of the three primaries vary with time $t$ and their distances to the libration point $\left(\xi_{0}, \eta_{0}\right)$ decrease with time. Therefore, we cannot determine the linear stability from ordinary method. So, we use the space-time inverse transformations of Meshcherskii [10] i.e. $x=\gamma^{-1 / 2} \xi, y=\gamma^{-1 / 2} \eta$. The positions of the primaries are fixed so that their distances to the libration points are invariable.

In phase-space, equations (10) may be written as

$$
\begin{aligned}
& \dot{\mathrm{u}}=\mathrm{u}_{1}, \quad \dot{\mathrm{v}}=\mathrm{v}_{1}, \\
& \dot{\mathrm{u}}_{1}-2 \mathrm{n} \mathrm{v}_{1}=(\mathrm{w} \xi \xi)_{0} \mathrm{u}+(\mathrm{w} \xi \eta)_{0} \mathrm{v}, \\
& \dot{\mathrm{v}}_{1}+2 \mathrm{nu}_{1}=\left(\mathrm{w}_{\eta} \xi\right)_{0} \mathrm{u}+\left(\mathrm{w}_{\eta \eta}\right)_{0} \mathrm{v},
\end{aligned}
$$

By using Meshcherskii [10] inverse transformations, and putting

$$
\begin{aligned}
& x^{\prime}=\gamma^{-1 / 2} u, y^{\prime}=\gamma^{-1 / 2} v, \\
& u^{\prime}=\gamma^{-1 / 2} u_{1}, v^{\prime}=\gamma^{-1 / 2} v_{1},
\end{aligned}
$$

In the matrix the system (12) can be written as follows:

$$
\left(\begin{array}{c}
\frac{\mathrm{dx}}{\mathrm{dt}} \\
\frac{\mathrm{dy^{ \prime }}}{\mathrm{dt}} \\
\frac{\mathrm{du}}{\mathrm{dt}} \\
\frac{d v^{\prime}}{\mathrm{dt}}
\end{array}\right)=\left(\begin{array}{cccc}
\frac{\alpha}{2} & 0 & 1 & 0 \\
0 & \frac{\alpha}{2} & 0 & 1 \\
(\mathrm{w} \xi \xi)_{0} & (\mathrm{w} \xi \eta)_{0} & \frac{\alpha}{2} & 2 \mathrm{n} \\
\left(w_{\eta \xi}\right)_{0} & (\mathrm{w} \eta \eta)_{0} & -2 \mathrm{n} & \frac{\alpha}{2}
\end{array}\right) \times\left(\begin{array}{c}
\mathrm{x}^{\prime} \\
\mathrm{y}^{\prime} \\
\mathrm{u}^{\prime} \\
\mathrm{v}^{\prime}
\end{array}\right)
$$

As the positions of the primaries are fixed and their distances to the libration points are invariable, the stability of (13) and (5) is consistent with each other. In fact, the original null solution when $\alpha_{1}=0$ has been disturbed into a non-trivial solution. Thus, the linear stability of this solution depends on the existence of stable region of the libration point, which in turn depends on the boundedness of the solution of linear and homogenous system of equations (13). We have determined the linear stability of the libration points. For this, we find the characteristic roots of the coefficient matrix of equation (13) numerically.

The characteristic equation of the coefficient matrix is

$$
\begin{aligned}
& \lambda^{4}-2 \alpha \lambda^{3}+\left(\frac{3}{2} \alpha^{2}+\mathrm{P}\right) \lambda^{2}-\left(\frac{1}{2} \alpha^{3}+\mathrm{P} \alpha\right) \lambda+ \\
& \left(\frac{1}{16} \alpha^{4}+\frac{1}{4} \mathrm{P} \alpha^{2}+\mathrm{Q}\right)=0,
\end{aligned}
$$

Where

$$
\mathrm{P}=4 \mathrm{n}^{2}-(\mathrm{w} \xi \xi)_{0}-\left(\mathrm{w}_{\eta \eta}\right)_{0}
$$

$\mathrm{Q}=(\mathrm{w} \xi \xi)_{0}\left(\mathrm{w}_{\eta \eta}\right)_{0}-(\mathrm{w} \xi \eta)_{0}^{2}$,

Where values of $\mathrm{w}_{\xi \xi}, \mathrm{w}_{\eta \eta}$ and $\mathrm{w}_{\xi \eta}$ are given by equations (11).

The characteristic roots of the equation (14) have been calculated at various libration points in the range $0<\gamma \leq 1,0 \leq \alpha_{1} \leq 1, \mu=0.019$. We have observed that there always exist seven liberation points in which three are asymptotically stable and four are unstable because we got at least one real characteristic root at each liberation points.

\section{Conclusion}

We have studied here the restricted four body problem with oblate primary and the infinitesimal mass is taken as variable. Bhatnagar [3] has taken the four body problem instead of the restricted three body problem and in these four bodies he has taken the three bodies (primaries) as spherical in shape which forms an equilateral triangular configuration. But in our problem we have taken oblate primary so we got an isosceles triangular configuration for the primaries (Eqn.-2). We have determined the equations of motion which are different from the equations of motions of Bhatnagar [3] by oblateness factor and variable mass parameters. Also we have determined the equilibrium points and found seven liberation points which are also different from Md Chand Asique et al. [8] because they have found eight liberation points. And we observed that out of seven liberation points, three are asymptotically stable (dark black in the tables) and four are unstable because we got at least one real characteristic root at each liberation points.

Our model is quite realistic and it has wider applications in this space age because most of the natural and artificial bodies moving in space are oblate bodies and losing their masses.

\section{Acknowledgement}

We are thankful to the Deanship of Scientific Research, College of Science in Zulfi, Majmaah University, Kingdom of Saudi Arabia, for providing all the research facilities.

\section{References}

[1] Abouelmagd, EI, Mostafa, A (2015) Out of plane equilibrium points locations and the forbidden movement regions in the restricted three-body problem with variable mass. Astrophys. Space Sci. 357:58, http://dx.doi.org/10.1007/s10509-015-2294-7.

[2] Baltagiannis, AN, Papadakis, KE (2011) Equilibrium points and their stability in the restricted four-body problem. International Journal of Bifurcation and Chaos 21, 2179, http://dx.doi.org/10.1142/s0218127411029707. 
[3] Bhatnagar, KB (1971) Periodic orbits of collision in the plane circular problem of four bodies. Indian Journal of Pure and Applied Math. Vol-2, No. - 4.

[4] Douskos, CN, Markellos, VV (2006) Out-of-plane equilibrium points in the restricted three body problem with oblateness. Astron. Astrophys. 446, 357-360. http://dx.doi.org/10.1051/00046361:20053828.

[5] Jeans, JH (1928) Astronomy and Cosmogony. Cambridge University Press, Cambridge.

[6] Khanna, M, Bhatnagar, KB (1999) Existance and stability of libration points in the restricted three body problem when the smaller primary is a triaxial rigid body and the bigger one and oblate spheroid. Indian Journal of Pure and Applied Math. 30(7), 721.

[7] McCuskey, SW (1963) Introduction to Celestial Mechanics. Addison-Wesley, Publishing Company, Inc, USA.

[8] Md. Chand Asique, et al. (a) (2015) On the R4BP when third primary is an oblate spheroid. Astrophys Space Sci. 357, 82 http://dx.doi.org/10.1007/s10509-015-2235-5.

[9] Md. Chand Asique, et al. (b) (2015) on the photogravitational $\mathrm{R} 4 \mathrm{BP}$ when third primary is an oblate/ prolate spheroid. Astrophys. Space Sci. 360, 13 http://dx.doi.org/10.1007/s10509-015-2522-1.

[10] Meshcherskii, I.V. (1949) Studies on the mechanics of bodies of variable mass. GITTL, Moscow.

[11] Mittal, A, et al. (2009) Periodic orbits generated by Lagrangian solutions of the restricted three body problem when one of the primaries is an oblate body. Astrophys. Space Sci 319, 63-73. http://dx.doi.org/10.1007/s10509-008-9942-0.

[12] Moulton, FR (1900) on a class of particular solutions of the problem of four bodies. Trans. Am. Math. Soc. 1, 17-29. http://dx.doi.org/10.1090/S0002-9947-1900-1500520-3.

[13] Shrivastava, AK, Ishwar, B (1983) Equations of motion of the restricted problem of three bodies with variable mass. Celest. Mech. 30, 323-328. http://dx.doi.org/10.1007/BF01232197.

[14] Singh, J, Ishwar, B (1984) Effect of perturbations on the location of equilibrium points in the restricted problem of three bodies with variable mass. Celest. Mech. 32 (4), 297-305. http://dx.doi.org/10.1007/BF01229086.

[15] Singh, J, Ishwar, B (1985) Effect of perturbations on the stability of triangular points in the restricted problem of three bodies with variable mass. Celest. Mech. 35, 201-207. http://dx.doi.org/10.1007/BF01227652.

[16] Singh, J (2003) Photogravitational restricted three body problems with variable mass. Indian Journal of Pure and Applied Math. 32 (2) 335-341.

[17] Singh, J \& Leke (2010) Stability of photogravitational restricted three body problem with variable mass, Astrophysics and Space Sci. 326 (2), 305-314 http://dx.doi.org/10.1007/s10509-009-0253-x.

[18] Szebehely, V (1967) Theory of orbits: The restricted Problem of Three Bodies. Academic Press, New York

[19] Zhang, M J, Zhao, C Y \& Xiong, Y Q (2012) on the triangular libration points in photo-gravitational restricted three body problem with variable mass. Astrophysics Space Sci. 337, 107-113, http://dx.doi.org/10.1007/s10509-011-0821-8. 